\title{
Range Minimum Queries in Minimal Space
}

\author{
Luís M. S. Russo \\ luis.russo@tecnico.ulisboa.pt \\ INESC-ID and Department of Computer Science and Engineering, \\ Instituto Superior Técnico, Universidade de Lisboa.
}

\begin{abstract}
We consider the problem of computing a sequence of range minimum queries. We assume a sequence of commands that contains values and queries. Our goal is to quickly determine the minimum value that exists between the current position and a previous position $i$. Range minimum queries are used as a sub-routine of several algorithms, namely related to string processing. We propose a data structure that can process these commands sequences. We obtain efficient results for several variations of the problem, in particular we obtain $O(1)$ time per command for the offline version and $O(\alpha(n))$ amortized time for the online version, where $\alpha(n)$ is the inverse Ackermann function and $n$ the number of values in the sequence. This data structure also has very small space requirements, namely $O(\ell)$ where $\ell$ is the maximum number active $i$ positions. We implemented our data structure and show that it is competitive against existing alternatives. We obtain comparable command processing time, in the nano second range, and much smaller space requirements.
\end{abstract}

Keywords: Range Minimum Queries, Union Find, Disjoint Sets, Bulk Queries, String Processing, Longest Common Extension.

\section{The Problem}

Given a sequence of integers, usually stored in an array $A$, a range minimum query (RMQ) is a pair of indexes $(i, j)$. We assume that $i \leq j$. The solution to the query consists finding in the minimum value that occurs in $A$ between the indexes $i$ and $j$. Formaly the solution is $\min \{A[k] \mid i \leq k \leq j\}$. There exist several efficient solutions for this problem, in this static offline context, see Section 5 . In this paper we consider the case where $A$ is not necessarially stored. Instead we assume that the elements of $A$ are streamed in a sequential fashion. Likewise we assume that the corresponding queries and are intermixed with the values of $A$ and the answers to the operations are computed online. Hence we assume that the input to our algorithm consists in a sequence of the following commands:

Value - represented by $\mathrm{V}$, is followed by an integer, or float, value $v$ and it indicates that $v$ is the next entry of $A$, i.e., $A[j]=v$.

Query - represented by Q, is followed by an integer that indicates a previous index of the sequence. The given integer corresponds to the element $i$ 
in the query. The element $j$ is the position of the last given value of $A$. Hence it is only necessary to specify $i$. This command can only be issued if an M command was given at position $i$ and no close command was given with argument $i$.

Mark - represented by M, indicates that future queries may use the current position $j$ as element $i$, i.e., as the beginning of the query.

Close - represented by $\mathrm{C}$, is also followed by an integer $i$ that represents an index of the sequence. This command essentially nullifies the effect of an M command issued at position $i$. Hence the command indicates that the input contains no more queries that use $i$. Any information that is being kept about position $i$ can be purged.

For simplicity we assume that the sequence of commands is not designed to hack our data structure. Hence we assume that no patological sequences are given as input. Examples of patological sequences would be: issuing the Mark command twice or more or mixed with Query; issuing a Close command for an index that was not marked; issuing Mark commands for positions that have been closed; etc.

Consider the following example sequence. We will use this sequence throughout the paper.

\section{22 M V 23 M V 26 M V 28 M V 32 M V 27 M V 35 M Q 4 C 3}

In this paper we study this type of sequences. Our contributions are the following:

- We propose a new algorithm that can efficiently process this type of input sequences. We show that our algorithm produces the correct solution.

- We analyze the algorithm and show that it obtains a fast running time and requires only a very small amount of space. Specifically the space requirements are shown to be at most $O(q)$, where $q$ is the number of queries. Recall that we do not store the array $A$. We further reduce this bound to $O(\ell)$. Consider at some instant the number of marked positions that have not yet been closed. We refer to these positions as active. The maximum number of active positions over all instants is $\ell$. The query time is shown to be $O(1)$ in the offline version of the problem and $O(\alpha(\ell))$ on the online version, where $\alpha$ is the inverse Ackermann function, see Theorem 2 and Corolary 1in Section 3.2. We also discuss the use of this data structure for real-time applications. We obtain a high probability $O(\log n)$ time for all operations, Theorem 3 . We also discuss trade-off that can reduce this bound to $O(\log \log n)$ for some operations, Theorem 4

- We implemented the online version of our algorithm and show experimentally that it is very efficient both in time and space.

\section{Data Structure Outline}

Let us now dicuss how to solve this problem, by gradually considering the challenge at hand. We start by describing a simple structure. We then proceed to 
improve its performance, first by selecting fast data structures which provide good time bounds and second by reducing the space requirements from $O(q)$ to $O(\ell)$.

Consider again the sequence in Section 1, Our first data structure is a stack, which we use in the same way as for building a Cartesian tree, see Crochemore and Russo [2020]. The process is simple. We start by pushing a $-\infty$ value into the stack, this value will be used as a sentinel. To start the discussion we will assume, for now, that every Value command is followed by a Mark command, meaning that every position is relevant for future queries.

An important invariant of this stack is that the values form an increasing sequence. Whenever a value is received it is compared with the top of the stack. While the value at hand is smaller the stack gets poped. At some point the input value will be larger than the top of the stack, even if it is necessary for the sentinel to reach the top. When the input value is larger than the top value it gets pushed into the stack. Another important property of this data structure is that the values in the stack are the only possible solutions for range minimum queries $(i, j)$, where $j$ is the current position of the sequence being processed and $i$ is some previous position.

To identify the corresponding $i$ it is usefull to keep, associated to each stack item, the set of positions that yield the corresponding item as the RMQ solution. Maintaining this set of positions is fairly simple. Whenever an item is inserted into the stack it is inserted with the current position. We number positions by starting at 1 . When an item is poped from the stack the set of positions associated to that item is transferred into the set of positions of the item below it. In our example the Value 27 command puts the positions 4 and 5 into the same set. The rightmost gray rectangle in Figure 1 illustrates the state of this data structure after processing the commands V $35 \mathrm{M}$ of our sample sequence. To process a Close command we remove the corresponding position from whatever set it belongs to, i.e., command $\mathrm{C}$ followed by $i$ removes $i$ from a position set.

Figure 1 illustrates the configuration of this data structure as it processes the following sequence of commands:

V 22 M V 23 M V 26 M V 28 M V 32 M V 27 M V 35 M Q 4 C 3

Each gray rectangle shows a different configuration. The leftmost configuration is obtained after the V $32 \mathrm{M}$ commands. The second configuration after the V $35 \mathrm{M}$ commands. The rightmost configuration is the final one after the C 3 . The solution to the Q 4 command is 27 , because it is the stack item associated with the position 4 in the rightmost configuration, these values are highlighted in bold.

Using a standard stack implementation it is possible to guarantee $O(1)$ time for the push and pop operations. Hence, ignoring the time required to process the sets of positions, the pairs of Value and Mark operations require only constant amortized time to compute. In the worst case a Value operation may need to discard a big stack, i.e., it may require poping $O(n)$ items, where $n$ is the total amount of positions in $A$. However since each operation executes at most one push operation the amortized time becomes $O(1)$. Hence the main challenge for this data structure is how to represent the sets of positions. To answer this question we must first consider how to compute the Query operation. Given this command, followed by a value $i$, we proceed to find the set that contains $i$ and report the corresponding stack element. For example to process the Q 4 


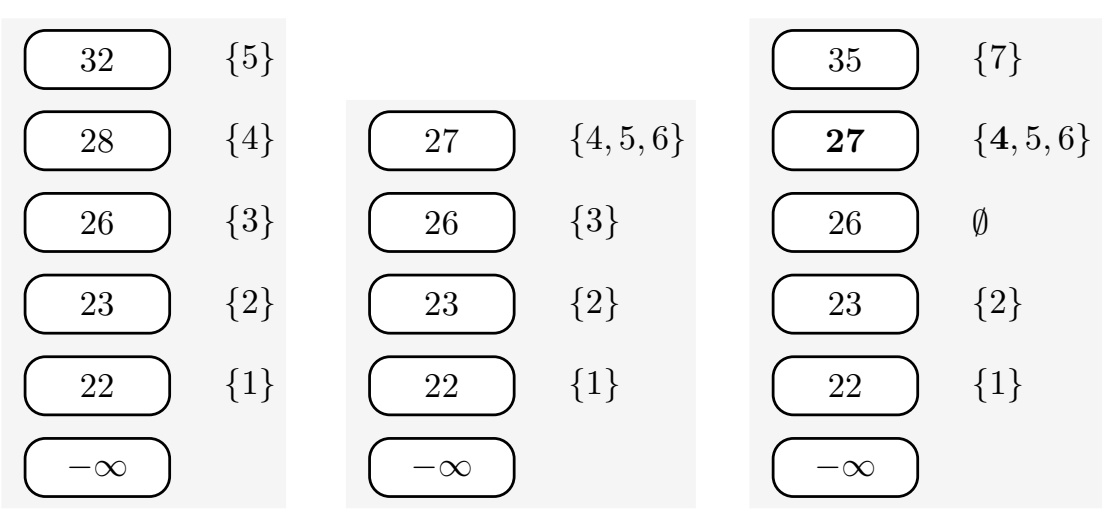

Figure 1: Illustration of structure configuration at different instances. Each gray rectangle shows the stack on the left and the corresponding sets of positions on the right.

command in the input sequence we most locate the set that contains position 4. In this case the set is $\{4,5,6\}$ and the corresponding element is 27 . Hence the essential operations that are required for the sets of positions are the union and the find operations. Union is used when merging sets in the Mark operation and find is used to identify sets in the Query operation.

A naive implementation requires $O(n)$ time for each operation. Instead we use a dedicated data structure that supports both operations in $O(\alpha(n))$ amortized time, where $\alpha(n)$ is the inverse Ackermann function. Note that although conceptually the Close command removes elements from the position sets this data structure is essentially ignoring these operations. They do not alter the Union-Find (UF) data structure. Hence, once an element is assigned to a set, it can no longer be removed. Fortunately the resulting procedure is still sound, albeit it requires more space. This version does require a large amount of space, specifically $O(n)$ space.

Let us now focus on reducing the space to $O(m)$, where $m$ is the total number of Mark commands, which should be equal to the total number of Close commands. We must also have that $m \leq q$, where $q$ is the number of Query commands, as there is no point in issuing redundant Mark commands. Note that $m$ may be much smaller than $n$ as there might be many more Value commands than Mark commands.

To guarantee that the size of the stack is at most $O(m)$ we now consider the situation where not all the Value commands are followed by Mark commands, otherwise $n$ and $m$ would be similar. In this case only the marked positions need to be stored in the stack, thus reducing its size. This separation of commands means that our operating procedure also gets divided. The Mark command only pushes elements into the stack. The Value commands only performs the poping commands. Hence in this scenario both the Mark and Value commands require $O(\alpha(n))$ amortized time.

To illustrate the division we have just described consider the following sequence of commands:

V 22 M V 23 V 26 M V 28 M V 32 M V 27 M V 35 M Q 4 C 3 


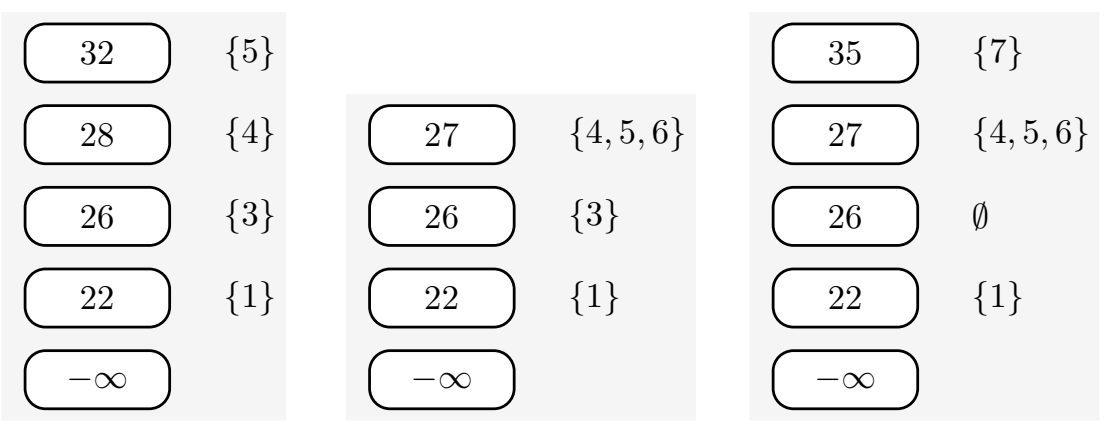

Figure 2: Illustration of structure configuration at different instances. In this sequence of commands there is no M command after V 23. Each gray rectangle shows the stack on the left and the corresponding sets of positions on the right.

We illustrate the state of the resulting data structure in Figure 2. Notice that in this sequence there is no $\mathrm{M}$ command after $\mathrm{V}$ 23. Therefore this value never gets inserted into the stack.

To reduce the size of the UF data structure we add a hash table to it. Without this table every one of the $n$ position values are elements for the UF data structure. Using a hash we can filter out only the marked positions. When a Mark command is issued we insert the current $j$ position as the hash key and the value is the current number of UF elements. This reduces the size of the UF data structure to $O(m)$. Moreover the hash table also requires only $O(m)$ space. Hence this data structure requires only $O(m)$ space and can process any sequence of commands in at most $O(\alpha(n))$ amortized time per command. When a Close $i$ command is issued we mark the position $i$ as deleted in the hash table, but we do not actually remove it from memory. The reason for this process is that a stack item might actually point to position $i$ and removing it would break the data structure. For the $O(m)$ space bound this is not an issue as inactive markings count for the overall total.

In the next section we discuss several nuances of this data structure, including how to further reduce the space requirements to $O(\ell)$ space and alternative implementations.

\section{The Details}

In this Section we will prove that the algorithm is correct and analyze its performance. We start of by giving a pseudo code description of the algorithms used for each command, Algorithms 3, 4, 5] and 6. In these algorithms we make some simplifying assumptions and use some extra commands that we will now define.

For simplicity we describe the data structure that does not use a hash-table. We use $S$ to represent the stack data structure, but we also use $S\left[k^{\prime}\right]$ to reference the element at position $k^{\prime}$. In general the top of the stack is at position $k$, which also corresponds to the number of elements in the stack. We use $k$ as a global variable. We also use $k$ as a bounded variable in the Lemma statements. Hence the value of $k$ must be derived from context. This is usually not a problem and 
in fact it is handy for the proofs, which most of the time only need to consider when $k$ is the top of the stack. We also use the notation $\operatorname{Top}(S)$ to refer to the top of the stack, this value is equal to $S[k]$. Note that this means that the element $S[k-1]$ is the one just below the Top element. Algorithms 11 and 2 used to manipulate the stack status and are given for completion. The set of positions associated with each stack item are denoted with the letter $P$. In our example we have that $P[4]=\{4,5,6\}$, see Figure 1.

In algorithm 3 we assume that the result of the Find command is directly a position index of $S$, therefore the expression $S[$ Find $(i)]$ for Algorithm 3 , The NOP command does nothing, it is used to highlight that without a hash table there is nothing for the Close command to execute.

The Make-Set function is used to create a set in the UF data structure, the first argument indicates the element that is stored in the set (position $j$ ) and the second argument the level of the last element on the stack $S$, i.e., $k$. It is the values given in this second argument that we expect Find to return. Likewise the Union function receives three arguments. The sets that we want to unite and again the top of the stack $k$. Note that in Algorithm 6 we use $\{j\}$ as one of the arguments to Union operation. In this case we are assuming that this operation makes the corresponding Make-Set operation.

Besides $k$ we have a few global variables, $j$ which indicates the current position in $A$ and $v$, which is not an argument of the Mark command but is used in that command. At that point it is assumed that $v$ is the last value given in the Value command.
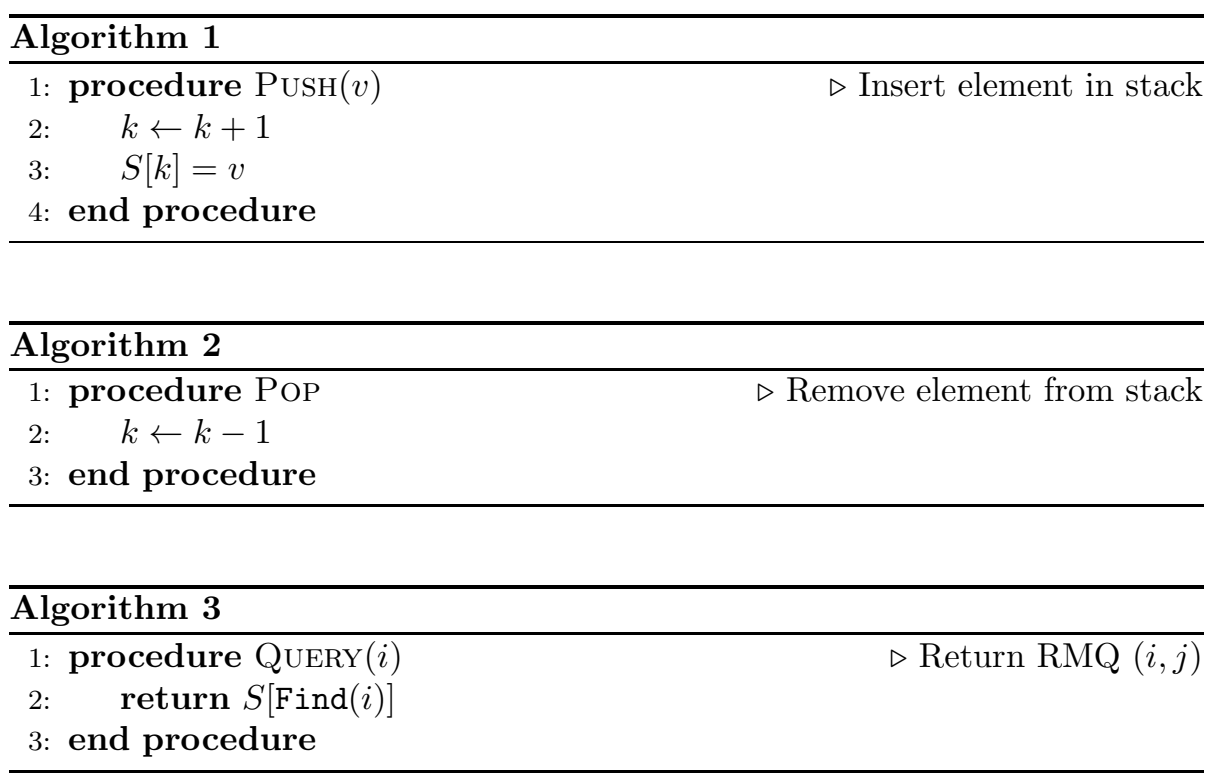

\subsection{Correctness}

In this Section we establish that our algorithm is correct, meaning the values obtained from our data structure actually correspond to the solutions of the given range minimum queries. We state several invariant properties that the structure always maintains. 


\begin{tabular}{ll}
\hline Algorithm 4 & \\
\hline 1: procedure CLOSE $(i)$ & $\triangleright$ Ignore command \\
2: NOP & \\
3: end procedure & \\
\hline
\end{tabular}
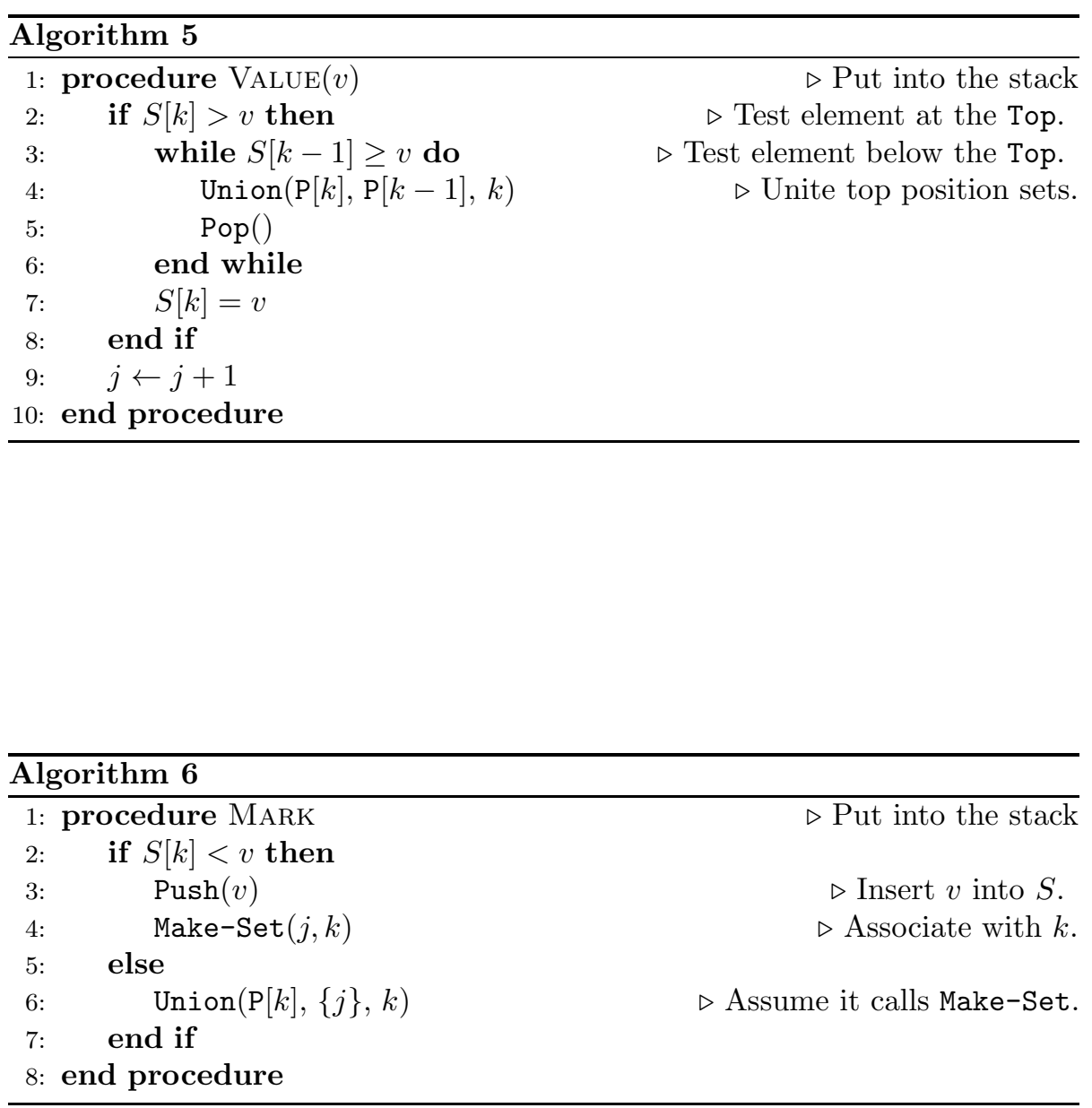
We consider the version of the data structure that consists of a stack and a UF structure. The version containing a hash is relevant for obtaining an efficient structure but does not alter the underlying operation logic. Hence the correctness of the algorithm is preserved, only its description is more elaborate.

We prove the invariant properties by structural induction, meaning that we assume that they are true before a command is processed and only need to prove that the property is maintained by the corresponding processing. For this kind of argument to hold it is necessary to verify that the given properties are also true when the structure is initialized, this is in general trivially true so we omit this verification from the following proofs. Another declutering observation is that the Query and Close commands do not alter our data structure and therefore are also omitted from the following proofs.

Let us start by establishing some simple properties.

Lemma 1. The stack $S$ always contains at least two elements.

Proof. In this particular proof it is relevant to mention the initial state of the stack $S$. The stack is initialized with two sentinel values, $-\infty$ followed by $+\infty$. Hence it initially contains at least two elements.

- The Mark command. This command does not uses the Pop operation and therefore never reduces the number of elements. The result follows by induction hypothesis.

- The Value command. For the Pop operation in line 5 of Algorithm 5 to execute the while guard in line 3 must be true. Note that when $k=2$ this guard consists in testing whether $-\infty=S[1]>v$, which is never the case and therefore a Pop operation is never executed in a stack that contains 2 elements.

Lemma 2. If $v$ was the argument of the last Value command and $k$ is the top level of that stack $S$ then $S[k] \leq v$.

Proof.

- The Mark command. When the if condition of Algorithm 6 is true we have that line 3 executes. After which $S[k]=v$ and the Lemma condition is verified. Otherwise the if condition is false and the stack is kept unaltered, in which case the result follows by induction hypothesis.

- The Value command. When the if condition of Algorithm 5 fails the Lemma property is immediate. Hence we only need to check the case when the if condition holds. In this case line 7 must eventually execute at which point we have that $S[k]=v$ and the Lemma condition is verified.

Let us now focus on more global properties. Next we show that the values stored in $S$ are in increasing order.

Lemma 3. For any indexes $k$ and $k^{\prime}$ of the stack $S$ we have that if $k^{\prime}<k$ then $S\left[k^{\prime}\right]<S[k]$. 
Proof.

- The Value command. This command does not push elements into the stack, instead it pops elements. This means that, in general, a few relations are discarded. The remaining relations are preserved by the induction hypothesis. The only change that we need to verify is if the Top of the stack $S$ changes, line 7 of Algorithm 5 . Hence we need to check the case when $k$ is the top level of the stack. Note that line 7 occurs immediately after the while cycle. Which means that its guard is false, i.e., we have that $S[k-1]<v=S[k]$. Hence the desired property was established for $k^{\prime}=k-1$. For any other $k^{\prime}<k-1$ we can use the induction hypothesis to conclude that $S\left[k^{\prime}\right]<S[k-1]$, which combined with the previous inequality and transitivity yields the desired property that $S\left[k^{\prime}\right]<S[k]$.

- The Mark command. The only operation performed by this command is to push the last element into the stack. Hence when $k$ is below the top of the stack the property holds by induction. Let us analyze the case when the top of the stack changes, i.e., when $k$ is the top level of the stack. The change occurs in line 3 of Algorithm $[$ in which case we have that $S[k-1]<v=S[k]$. Hence we extend the argument for $k^{\prime}<k-1$ as in the Value command by induction hypothesis and transitivity.

Likewise the converse of this Lemma can now be established.

Lemma 4. For any indexes $k$ and $k^{\prime}$ of the stack $S$ we have that if $S[k]<S\left[k^{\prime}\right]$ then $k<k^{\prime}$.

Proof. Assume by contradiction that there are $k$ and $k^{\prime}$ such that $S[k]<S\left[k^{\prime}\right]$ and $k^{\prime} \leq k$. Because $S[k] \neq S\left[k^{\prime}\right]$ we have that $k \neq k^{\prime}$, since we are using $S$ as an array. Hence we must have that $k^{\prime}<k$ and can now apply Lemma 3 to conclude that $S\left[k^{\prime}\right]<S[k]$, which contradicts the order relation in our hypothesis.

This sorted property also gives structure to the sets of positions.

Lemma 5. For any indexes $k^{\prime}<k$ and positions $p^{\prime} \in P\left[k^{\prime}\right]$ and $p \in P[k]$ we have that $p^{\prime}<p$.

Proof.

- The Mark command. This operation inserts the current position $j$ into the set that corresponds to the top of the stack. The top might have been preserved or created by the operation, both cases can be justified in the same way. We only need to consider the case when $\operatorname{Top}(S)=S[k]$ and $p=j$, any other instanciation of the variables in the Lemma will correspond to relations that were established before the structure was modified. Hence we only need to show that $p^{\prime}<j$ for any $p^{\prime}$ in any $P\left[k^{\prime}\right]$. This is trivial because $j$ represents the current position in $A$, which is therefore larger than any previous position of $A$ that may be represented by $p^{\prime}$. 
- The Value command. As this command pops elements from the stack, it has the side effect of merging the position sets. Hence the only new relation is for positions at the top of the stack, i.e., when $p \in P[k]$ and $\operatorname{Top}(S)=S[k]$. We only need to consider where position $p$ was before the operation, i.e., $p \in P_{b}\left[k_{b}\right]$, were $P_{b}\left[k_{b}\right]$ represents a set of positions before the operation is executed. Because the Value command merges the position sets which are highest on the stack we have that $k \leq k_{b}$. Now, for any $k^{\prime}<k$ and $p^{\prime} \in P\left[k^{\prime}\right]$, we have that $P\left[k^{\prime}\right]=P_{b}\left[k^{\prime}\right]$ because the sets of positions below the top of the stack are not altered by the operation. In essence we have that $k^{\prime}<k_{b}$ and $p^{\prime} \in P_{b}\left[k^{\prime}\right]$ and $p \in P_{b}\left[k_{b}\right]$, therefore by induction hypothesis we obtain $p^{\prime}<p$, as desired.

We can now state our final invariant, which establishes that our algorithm is correct.

Theorem 1. At any given instant when $j$ is the current position over $A$ we have that if $i \in P\left[k^{\prime}\right]$ then $R M Q(i, j)=S\left[k^{\prime}\right]$.

Proof.

- The Mark command. This command does not alter the sequence $A$. Therefore none of the $\operatorname{RMQ}(i, j)$ values change. Since almost all positions and position sets $P\left[k^{\prime}\right]$ are preserved the implication is also preserved. The only new position is $j \in P[k]$, therefore the only case we need to consider is when $i=j$ and $k^{\prime}$ is the top level of the stack $S$, i.e., $k^{\prime}=k$. In this case we have that $\operatorname{RMQ}(j, j)=A[j]=v$, where $v$ is the argument given in the last Value command. Now let us consider the if condition in line 2 of Algorithm 6. This further divides the argument into two cases:

- When this condition holds then line 3 of Algorithm [6] executes and makes $S[k]=v$. Hence $\operatorname{RMQ}(j, j)=S[k]$.

- When this condition fails we have $v \leq S[k]$. Applying Lemma 2 we obtain $S[k] \leq v$ and therefore conclude that $S[k]=v$. Hence $\operatorname{RMQ}(j, j)=S[k]$.

- The Value command. This command essentially adds a new value $v$ at the end of $A$, i.e., it sets $A[j]=v$, where $j$ is now the last position of $A$. This implies that $j$ is not yet a marked position. Therefore for this command we do not need to consider $i=j$ because $j$ is not a member of a position set $P\left[k^{\prime}\right]$.

Thus we only need to consider cases when $i<j$. Consider such an index $i$, which moreover belongs to the position set $P\left[k^{\prime}\right]$, i.e., $i \in P\left[k^{\prime}\right]$. The position $i$ must necessarily occur in some set $P_{b}\left[k_{b}^{\prime}\right]$, which is a set of positions that exists before the Value operation alters the stack. In this case we have by induction hypothesis that $\operatorname{RMQ}(i, j-1)=S_{b}\left[k_{b}^{\prime}\right]$. We now divide the proof into two cases:

- When $S_{b}\left[k_{b}^{\prime}\right] \leq v$, in which case $\operatorname{RMQ}(i, j)=S_{b}\left[k_{b}^{\prime}\right]$. In this case we only need to show that the Value command does not alter the 
index $k_{b}^{\prime}$ of the stack, i.e., that $i \in P\left[k_{b}^{\prime}\right]$ and that $S_{b}\left[k_{b}^{\prime}\right]=S\left[k_{b}^{\prime}\right]$. Therefore the desired property holds for $k^{\prime}=k_{b}^{\prime}$. This is imediate as the case hypothesis means that even if the Value operation happens to extrude level $k_{b}^{\prime}$ to the top of the stack it does eliminate it, because Lemma 3 implies that $S_{b}\left[k_{b}^{\prime}-1\right]<S_{b}\left[k_{b}^{\prime}\right] \leq v$, and therefore the while guard in line 3 fails.

- When $v<S_{b}\left[k_{b}^{\prime}\right]$, in which case $\operatorname{RMQ}(i, j)=v$. In this case the value $S_{b}\left[k_{b}^{\prime}\right]$ will be discarded by the Value command. Let $k$ correspond to the level that is at the top of the stack, after the command. By Lemma 2 we have that $S[k] \leq v$ combining both these inequalities yields $S[k]<S_{b}\left[k_{b}^{\prime}\right]$. Using Lemma 3 we have that $S[k-1]<S[k]$, note that Lemma 1 guarantees that the level $k-1$ exists. Moreover because $k$ is the top level of $S$ after the command we have $S_{b}[k-1]=$ $S[k-1]$. Combining these relations we obtain that $S_{b}[k-1]<S_{b}\left[k_{b}^{\prime}\right]$, to which we apply Lemma 4 , to conclude that $k-1<k_{b}^{\prime}$. Therefore either $k=k_{b}^{\prime}$ or the level $k_{b}^{\prime}$ was excluded from the stack. In both cases position $i$ must be in $P[k]$, either because it was already there or it was eventually transferred by the union commands in line 4. Hence we only need to check that $S[k]=v$. Let $k_{b}$ be the Top of stack $S_{b}$ before the command is executed. Hence $k_{b}^{\prime} \leq k_{b}$ and by Lemma 3 we obtain $S_{b}\left[k_{b}^{\prime}\right] \leq S_{b}\left[k_{b}\right]$. Using this case hypothesis and transitivity we obtain that $v<S_{b}\left[k_{b}\right]$. This implies that the condition of the if in line 2 of Algorithm $[5$ is true. Therefore line 7 eventually executes and obtains the condition $S[k]=v$ as desired.

\subsection{Analysis}

In this section we discuss several issues related to the performance of our data structure. Namely we start off by reducing the space requirements from $O(m)$ to $O(\ell)$. First we need to notice in which ways our data structure can waist space. In particular the Close command waists space in the stack itself. In the rightmost structure of Figure 11 we have that the set $P[3]$ becomes empty after the C 3 command. This set which corresponds to $S[3]=26$ on the stack. In essence the item $S[3]$ is no longer necessary in the stack. However it is kept inactive in the stack, the hash table and the UF data structure. It is marked as inactive in the hash table, but it still occupies memory.

Recall that our data structure consists of three components: a stack, a hash table and a Union-Find data structure. These structures are linked as follows: the stack contains values and pointers to the hash table; the hash-table uses sequence positions as keys and UF elements as values; the Union-Find data structure is used to manipulate sets of reduced positions and each set in turn points back to a stack position.

Let us now use an amortizing technique to bound the space requirements of this structure. We start off by allocating a data structure that can contain at most $a$ elements, where $a$ is a small initial constant. Allocating a structure with this value implies the following guarantees:

- It is possible to insert $a$ elements into the stack without overflow. 
- It is possible to insert $a$ elements into the hash table and the overall occupation is always less than half. This guarantees average and high probability efficient insertions and searches.

- It is possible to use $a$ positions for Union-Find operations.

Hence we can use this data structure until we reach the limit $a$. When the limit is reached we consider the number of currently active marked positions, i.e., the number of positions $i$ such that $\mathrm{M}$ was issued at position $i$, but up to the current position no Close $i$ was never issued. To determine this value it is best to keep a counter $c$. This counter is increased when a Mark command is issued, unless the previous command was also a Mark command, in which case it is a repeated marking for a certain position. The counter is decreased when a Close $i$ is issued, provided position $i$ is currently active, i.e., it was activated by some Mark command and it has not yet been closed by any other Close command. Hence by consulting this counter $c$ we can determine in $O(1)$ time the number of active positions at this instant. We can now alloc a new data structure with $a^{\prime}=2 c$, i.e., a data structure that can support twice as many elements as the number of current active positions. Then we transfer all the active elements from the old data structure to the new data structure. The process is fairly involved, but in essence it requires $O(a \times \alpha(a))$ time and when it finishes the new data structure contains all the active positions, which occupy exactly half of the new data structure. This factor is crucial as it implies that the amortized time of this transfer is in fact $O(\alpha(a))$ and moreover that the allocated size is at most $O(2 \ell)$.

We now describe how to transfer only the active elements from the old data structure to the new data structure. First we mark all the elements in the old stack as inactive. In our implementation we make all the values negative, as the test input sequences contained no negative values but other marking schemes may be used. This is also the scheme we used to mark inactive hash entries.

Now traverse the old hash table and copy all the active values to the new hash table. Also initilize the pointers from the new hash table to the new UF data structure. The new UF positions are initialized incrementally, starting at 1. Hence every insertion into the new hash function creates a new UF position, that is obtained incrementally from the last one. We also look up the old UF positions that are given by active entries of the old hash table. We use those old active sets to reactivate the old stack entries. This process allowed us to identify which stack entries are actually relevant in the old stack. With this information we can compact the old stack by removing the inactive positions. We compact the old stack directly to the new stack, so the new stack contains only active positions. We also add pointers from the old stack to the new stack. Each active entry of the old stack points to its correspondent in the new stack. In our implementation this was done by overriding the pointers to the old hash table, as they are no longer necessary.

At this point the new stack contains the active values, but it still has not initialized the pointers to the new hash table. These pointers are in fact position values, because positions are used as keys in the hash-table. To initialize these pointers we again traverse the active entries of the old hash table and map them to the old UF positions and to the corresponding old stack items. We now use the pointer from the old stack item to the new stack item and update the position pointer of the new stack to the key of the active entry of the new 
hash that we are processing. This assignment works because positions are kept invariant from the old data structure to the new one. Therefore these positions are also keys of the new hash. We finish this process by updating the pointers of the new UF data structure to point to the corresponding items of the new stack. Since we now know the active items in the new stack and have pointers from the new stack to the new hash and from the new hash to the new UF position, we can simply assign the link from the new UF set back to the item of the new stack item. Thus closing this reference loop.

At this point almost all of the data structure is linked up. The new stack points to the new hash table, the new hash table points to the new UF structure and the sets of the new UF structure point to the new stack. The only missing ingredient is that the sets of the new UF structure are still singletons, because no Union operations have yet been issued. The main observation to recover this information is that several positions in the new UF structure point to the same item in the new stack. Those positions need to be united into the same set. To establish these unions we traverse the new UF data structure. For each UF position we determine its corresponding stack item, note that this requires a Find operation. We then follow its pointer to an item in the new hash, and a pointer from that item back to a position in the new UF data structure. Now we unite two UF sets, the one that contained the initial position and the one that contains the position that was obtained by passing through the stack and the hash.

Theorem 2. It is possible to process online a sequence of $R M Q$ commands in $O(\ell)$ space using $O(\alpha(\ell))$ expected amortized time per command.

Proof. The discussion in this section essentially establishes this result. We only need to point out the complexities of the data structures that we are using. As mentioned before the UF structure requires $O(\alpha(n))$ amortized time. The stack is implemented over an array and therefore requires $O(1)$ per Push and Pop command. In theory we consider a hash-table with separate chaining and amaximum load factor of $50 \%$, which obtains $O(1)$ expected time per operation. In practice we implemented a linear probing approach.

The final argument is to show that the transfer process requires $O(\alpha(\ell))$ amortized time. Whenever a transfer process terminates the resulting structure is exactly half full. As the algorithm progresses elements are inserted into the structure until it becomes full. Whenever an element is inserted we store 2 credits. Hence when the structure is full there is a credit for each element it contains, therefore there are enough credits to amortize a full transfer process. We assume that these credits are actually multiplied by $\alpha(\ell)$ and whatever is the constant of the transfer procedure is.

One important variation of the above procedure is the offline version of the problem. Meaning that we are given the complete sequence of commands and are allowed to process them as necessary to obtain better performance. In this case we can use a more efficient variant of the Union Find data structure and obtain $O(1)$ time per operation, proposed by Gabow and Tarjan [1985].

Corolary 1. It is possible to process offline a sequence of $R M Q$ commands in $O(\ell)$ space using $O(1)$ expected amortized time per command. 
On the other extreme of applications we may be interrested in real time applications. Meaning that we need to focus on minimizing the worst case time that is necessary to process a given command. In this case we can modify our data structure to avoid excessively long operations, i.e., obtain stricter bounds for the worst case time. As an initial result let us de-amortize the transfer procedure, assuming the same conditions as in Theorem 2 .

Lemma 6. Given a sequence of $R M Q$ commands it is possible to processes them so that the transfer procedures require an overhead of $O(\alpha(\ell))$ expected amortized time per command.

Proof. Note that the transfer process requires $O(a \times \alpha(a))$ amortized time to transfer a structure that supports $a$ elements.

We modify the transference procedure so that it transfers two full structures at the same time, by merging their active elements into a new structure. The process is essentially similar to the previous transference procedure, with a few key differences.

An element can only be considered active if it is not marked as inactive in one of the old hashes. More precisely: if it is marked as active in one hash and as inactive in the other then it is inactive; if it is marked as active in one hash and does not exists in the other then it is active; if it is marked as active in both then it is active.

Once the active elements of the old stacks are identified they are merged into the new stack, by using the same merging procedure that is used in mergeSort algorithm, with the proviso that there should be only one copy of the sentinel in the merged stack. The third important sincronization point is the union commands. Before starting this process it is necessary that all the information from the old structures has been transfered to the new one, recall that this process generaly iterates over the new structure, not the old ones.

When the old structures can support $a_{1}$ and $a_{2}$ elements respectively the merging process requires $O\left(a_{1}+a_{2}\right)$ operations. Note that we do not mean time, instead we mean primitive operations on the data structures that compose the overall structure, namely accessing the hash function, following pointers or calling union or find. Given this merging primitive we can now deamortize our transfer process. Instead of immediately discarding a structure that hits its full occupancy we keep it around because we can not afford to do an immediate transfer. Instead when we have at least two full structures we initiate the transfer process. Again to avoid exceeding real time requirements this process is kept running in parallel, or interleaved, with the processing of the remaining commands in the sequence. Since this procedure requires $O\left(a_{1}+a_{2}\right)$ operations, it is possible to tune it to guarantee that it is terminated by the time that at most $\left(a_{1}+a_{2}\right) / 2$ commands are processed. In this case each command only needs to contribute $O(1)$ operations to the merging process. Each operation requires has an expected $O(\alpha(\ell))$ time, which yields the claimed value.

Hence, at any given instant, we can have several structures in memory. In fact we can have at most four, which serve the following purporses:

- One active structure. This structure is the only one that is currently active, meaning that it is the only structure that still supports Mark and Value commands.

- Two static full structures that are currently being merged. 
- One destination structure that will store the result of the merged structures. In general this structure is in some inconsistent state and does not process Query commands. The only command that it accepts is Close.

At any point of the execution some or all of the previous structures may be in memory. The only one that is always guaranteed to exist is the active structure. Now let us discuss how to process commands with these structures.

- The Query command is processed by all structures, except the destination structure which is potentially inconsistent. From the three possible values we return the overall minimum. In this case we are assuming that if the query position $i$ is smaller than the minimum position index stored in the structure than it returns its minimum value, i.e., the value above the $-\infty$ sentinel.

- The Mark and Value commands modify only the active structure.

- The Close command is applied to all the structures, including the destination structure. This causes no conflict or inconsistency. Recall that elements are not removed from the hashes, they are only marked as inactive.

If we have only the active structure in memory, we use it to process the Mark and Value commands. When this active structure gets full we mark it as static and ask for a new structure that supports the same number $a$ of elements. This structure becomes the new active structure. Note that requesting memory may require $O(a)$ time, assuming we need to clean it. This can be mitigated by using approaches as Briggs and Torczon 1993 or assuming that this process was previously executed, which is possible with in our approach.

As soon as the second structure becomes full we start the merging process to a new destination structure. We consult the number of active elements in each one, $c_{1}$ and $c_{2}$. We request the destination structure to support exactly $c_{1}+c_{2}$ elements. This implies that once the merge procedure is over the destination structure is full and no further elements can be inserted into it. At which point we need to request another active structure. If the full structures have sizes $a_{1}$ and $a_{2}$ we ask for an active structure that can support $\left(a_{1}+a_{2}\right) / 2$ elements. As argued above this active structure only gets full after the merging process finishes. At that point the original full structures can be discarded and again we have two full structures, the result of the previous merger and the filled up active structure. At this point we repeat the process.

The reason to have a division by 2 associated with $a_{1}+a_{2}$ is that its iteration yields a geometric series that does not exceed $2 \ell$. Hence implying that none of the structures need to support more that $2 \ell$ elements. This can also be verified by induction. Assuming that the original alloc size $a$ is also less than $2 \ell$, we have by induction hypothesis that $a_{1} \leq 2 \ell$ and $a_{2} \leq 2 \ell$ therefore $\left(a_{1}+a_{2}\right) / 2 \leq$ $(2 \ell+2 \ell) / 2 \leq 2 \ell$. Also by the definition of $\ell$ we also have that $c_{1} \leq \ell$ and $c_{2} \leq \ell$ which implies that the destination structures also support at most $2 \ell$ elements. Since the algorithm uses a at most 4 structures simultaneously, we can thus conclude that the overall space requirements of the procedure is $O(\ell)$.

Note that in the worst case the time bound of the UF structures is $O(\log \ell)$ rather than $O(\alpha(\ell))$. Also note that using a strict worst case analysis would yield 
an $O(\ell)$ worst case time for our complete data structure. Because it contains a hash-table. To avoid this pathological analysis we instead consider a high probability upper bound. In this context we obtain an $O(\log \ell)$ time bound with high probability, for all commands except the Value command. Hence let us now address this command.

Theorem 3. It is possible to process, in real time, a sequence of $R M Q$ commands in $O(\ell)$ space and in $O(\log \ell)$ time per operation with high probability.

Proof. Given the previous observations we can account $O(\log \ell)$ time for the UF structure and the hash table, with high probability, see Mitzenmacher and Upfal [2017]. Lemma 6 de-amortized the transfer operation, hence in this proof we only need to explain how to de-amortize the Value operation.

Algorithm 5 specifies that given an argument $v$ this procedure removes from the stack $S$ the elements that are strictly larger than $v$. This process may end up removing all the elements from the stack, except obviously the $-\infty$ sentinel. Hence its worst case time is $O(m)$, where $m$ is the maximum number of elements in the stack. The transfer procedure guarantees that the stack does not accumulate deactivated items and therefore we have that $m=O(\ell)$. This is still too much time for a real time operation. Instead we can replace this procedure by a binary search over $S$, i.e., we assume that stack is implemented on an array and therefore we have direct access to its elements in constant time. As shown in Lemma 3 the elements of $S$ are sorted. Therefore we can compute a binary search for the position of $v$ and discard all the elements in $S$ that are larger than $v$ in $O(\log \ell)$ time. Recall that we use variable $k$ to indicate the top of the stack. Once the necessary position is identified we update $k$.

However Algorithm [5] also specifies that each element that is removed from the stack invokes a Union operation, line 4. To perform these unions in real time we need a different UF data structure.

Most UF structures work by choosing a representative element for each set. The representative is the element that is returned by the Find operation. This representative is usually an element of the set it represents. The representative either posseses, or is assigned, some distinct feature that makes it easy to identify. In the UF structure by Tarjan and van Leeuwen [1984] a representative is stored at the root of a tree.

Lemma 5 essentially states that the sets that we are interrested in can be sorted, without incosistencies among elements of diferent sets. Hence this provides a natural way for choosing a representative. Each set can be represented by its minimum element. With this representation the Find $(p)$ operation consists in finding the largest representative that is still less than or equal to $p$, i.e., the Predecessor. The Union operation simply discards the largest representative and keeps the smallest one. Hence we do not require an extra data structure, it is enough to store the minimums along with values within the stack items. To compute the Predecessors we perform a binary search over the minimums. This process requires $O(\log \ell)$ time. Moreover the variable $k$ allows us to perform multiple Union operations at once. Let us illustrate how to use this data structure for our goals. Recall the sample command sequence:

V 22 M V 23 M V 26 M V 28 M V 32 M V 27 M V 35 M Q 4 C 3 


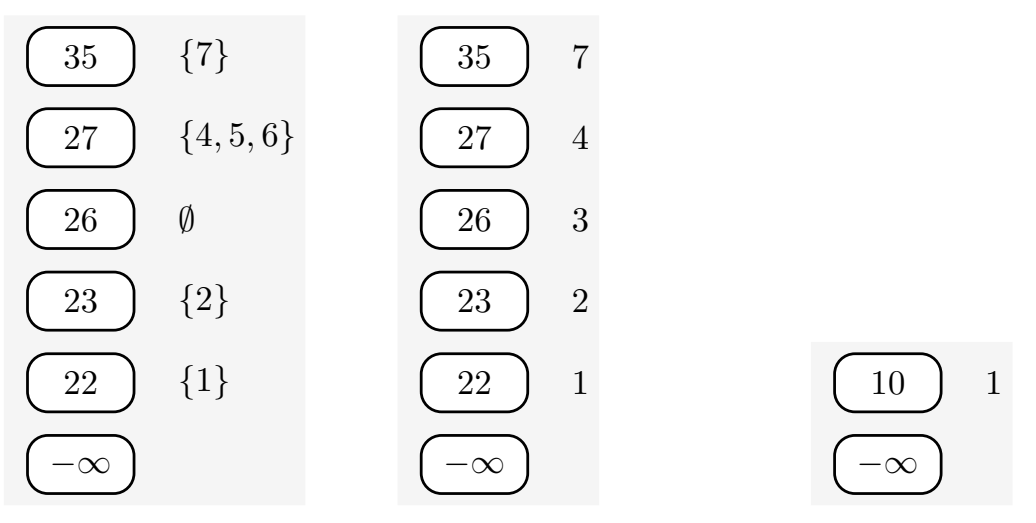

Figure 3: Illustration structure configuration using minimums to represent position sets.

Now assume that after this sequence we also execute the command V 10. We illustrate how a representation based on minimums processes these commands, Figure 3. The structure on left is the configuration after the initial sequence of commands. The structure in the middle represents the actual configuration that is stored in memory. Note that for each set we store only its minimum element. In particular note that the set associated with value 26 is represented by 3 , even though position 3 was already marked as closed. As mentioned the hash-table keeps track of which positions are still open and closed positions are removed during transfer operations. This means that until then it is necessary to use all positions, closed or not, for our UF data structure. Hence the representative of a set is the minimum over all positions that are related to the set, closed or not. The structure on the right represents the structure after processing the $\mathrm{V} 10$ command.

Note that in this final configuration the set, of active positions, associated with value 10 should be $\{1,2,4,5,6,7\}$. However it is represented only by the value 1 . This set should be obtained by the following sequence of Union operations $\{1\} \cup\{2\} \cup\{4,5,6\} \cup\{7\}$. This amounts to removing the numbers 2,4 and 7 , which is obtained automatically when we alter the variable $k$.

Summing up, our data structure consists of the following elements:

- An array storing stack S. Each element in the stack contains a value $v$ and position $i$, which is the minimum of the position set it represents.

- A hash-table to identify the active positions. In this configuration no mapping is required, it is enough to identify the active positions.

The general procedure for executing commands and the respective time bounds are the following:

- The Value command needs to truncate the stack, by updating variable $k$. This process requires $O(\log \ell)$ time because of the binary search procedure, but it can actually be improved to $O(1+\log d)$ time where $d$ is the number of positions removed from the position tree, by using an exponential search that starts at the top of the stack. Using an exponential search the expected amortized time of this operation is $O(1)$. 
- The Mark command needs to add an element to the hash-table and an element to the stack $S$. This requires $O(\log \ell)$ time with high probability. The Make-Set or Union operations require only $O(1)$ time hence the overall time is dominated by $O(\log \ell)$. The expected time of this operation is $O(1)$.

- The Query command needs to search for an element in the hash-table and compute a Find operation. The Find operation is computed with a binary search over minimums stored in the items of the stack. This operation requires $O(\log \ell)$ time with high probability. The expected amortized time is also $O(\log \ell)$, but it can be improved to $O(1+\log (j-i+1))$ for a query with indexes $(i, j)$, by using an exponential search from the top of the stack.

- The Close command needs to remove an element from the hash-table. This requires $O(\log \ell)$ time with high probability and $O(1)$ expected time.

The data structure of the previous theorem is simple because most of the complex de-amortizing procedure is handled in Lemma 6. We now focus on how to further reduce the high probability time bounds to $O(\log \log n)$. A simple way to obtain this is to have $\ell=O(\log n)$, i.e., having at most $O(\log n)$ active positions at each time. This may be achieved if Query positions are not necessarily exact, meaning that the data structure actually returns the solution for a query $\left(i^{\prime}, j\right)$ instead of $(i, j)$. The goal is that $j-i$ is similar in size of $j-i^{\prime}$. Meaning that $j-i \leq j-i^{\prime}<2(j-i)$. In this scenario it is enough to keep $O(\log n)$ active positions, i.e., positions $i^{\prime}$ for which $j-i^{\prime}=2^{c}$ for some integer $c$. Since the data structure of Theorem 3 does not use the hash-table to reduce the position range, we can bypass its use in these queries. It is enough to directly determine the predecessor of $i$ among the minimums stored in the stack $S$. Which is computed with a binary search or exponential search as explained in the proof.

The problem with this specific set of positions is that when $j$ increases the active positions no longer provide exact powers of two. This is not critical because we can adopt an update procedure that provides similar results. Let $i_{1}<i_{2}<i_{3}$ represent three consecutive positions that are currently active. When $j$ increases we check whether to keep $i_{2}$ or discard it. It is kept if $j-i_{1}>$ $2\left(j-i_{3}\right)$, otherwise it is discarded. Hence we keep a list of active positions that gets updated by adding the new position $j$ and checking two triples of active positions. We keep an index that indicates which triple to check and at each step use it to check two triples, moving from smaller to larger position values. The extremes of the list are not checked. We show the resulting list of positions in Table 1, where the bold numbers indicate the triples that will be checked in the next iteration. Whenever the triples to check reach the end of the list we have that the size of the list is at most $2 \log _{2} n$, because the verification guarantees that the value $j-i$ is divided in half for every other position $i$. Therefore it takes at $\operatorname{most}_{2} \log _{2} n$ steps to traverse the list. Hence this list can contain at most $4 \log _{2} n=O(\log n)$ positions and each time $j$ is updated only $O(1)$ time is used. 


$\begin{array}{rrrrrrrrrr}\mathbf{1} & & & & & & & & & \\ \mathbf{1} & \mathbf{2} & & & & & & & & \\ \mathbf{1} & \mathbf{2} & \mathbf{3} & & & & & & & \\ \mathbf{1} & \mathbf{2} & \mathbf{3} & 4 & & & & & & \\ 1 & \mathbf{3} & \mathbf{4} & \mathbf{5} & & & & & & \\ \mathbf{1} & \mathbf{3} & \mathbf{4} & 5 & 6 & & & & & \\ 1 & \mathbf{4} & \mathbf{5} & \mathbf{6} & 7 & & & & & \\ 1 & 4 & \mathbf{6} & \mathbf{7} & \mathbf{8} & & & & & \\ \mathbf{1} & \mathbf{4} & \mathbf{6} & 7 & 8 & 9 & & & & \\ 1 & \mathbf{4} & \mathbf{7} & \mathbf{8} & 9 & 10 & & & & \\ 1 & 4 & \mathbf{7} & \mathbf{9} & \mathbf{1 0} & 11 & & & & \\ 1 & 4 & 7 & 9 & \mathbf{1 0} & \mathbf{1 1} & \mathbf{1 2} & & & \\ \mathbf{1} & \mathbf{4} & \mathbf{7} & 9 & 10 & 11 & 12 & 13 & & \\ 1 & \mathbf{7} & \mathbf{9} & \mathbf{1 0} & 11 & 12 & 13 & 14 & & \\ 1 & \mathbf{7} & \mathbf{1 1} & \mathbf{1 2} & 13 & 14 & 15 & & & \\ 1 & 7 & \mathbf{1 1} & \mathbf{1 3} & \mathbf{1 4} & 15 & 16 & & & \\ 1 & 7 & 11 & \mathbf{1 4} & \mathbf{1 5} & \mathbf{1 6} & 17 & & & \\ 1 & 7 & 11 & 14 & \mathbf{1 6} & \mathbf{1 7} & \mathbf{1 8} & & & \\ \mathbf{1} & \mathbf{7} & \mathbf{1 1} & 14 & 16 & 17 & 18 & 19 & & \\ 1 & 7 & \mathbf{1 1} & \mathbf{1 4} & \mathbf{1 6} & 17 & 18 & 19 & 20 & \\ 1 & 7 & 11 & \mathbf{1 6} & \mathbf{1 7} & \mathbf{1 8} & 19 & 20 & 21 & \\ 1 & 7 & 11 & \mathbf{1 6} & \mathbf{1 9} & \mathbf{2 0} & 21 & 22 & & \\ 1 & 7 & 11 & 16 & \mathbf{1 9} & \mathbf{2 1} & \mathbf{2 2} & 23 & & \\ 1 & 7 & 11 & 16 & 19 & 21 & \mathbf{2 2} & \mathbf{2 3} & \mathbf{2 4} & \\ \mathbf{1} & \mathbf{7} & \mathbf{1 1} & 16 & 19 & 21 & 22 & 23 & 24 & 25 \\ 1 & \mathbf{1 1} & \mathbf{1 6} & \mathbf{1 9} & 21 & 22 & 23 & 24 & 25 & 26 \\ 1 & 11 & \mathbf{1 9} & \mathbf{2 1} & \mathbf{2 2} & 23 & 24 & 25 & 26 & 27 \\ 1 & 11 & \mathbf{1 9} & \mathbf{2 3} & \mathbf{2 4} & 25 & 26 & 27 & 28 & \\ 1 & 11 & 19 & \mathbf{2 4} & \mathbf{2 5} & \mathbf{2 6} & 27 & 28 & 29 & \\ 1 & 11 & 19 & \mathbf{2 4} & \mathbf{2 7} & \mathbf{2 8} & 29 & 30 & & \\ & & & & & & & & & \end{array}$

Table 1: Sequence of active position lists

Another alternative for obtaining $O(\log \log n)$ high probability time is to change the UF structure. In this case we use the same approach as Theorem 3 that relies on predecessor searches to compute the Find operation. This time we consider the Van Emde Boas tree that supports this operation efficiently, but requires longer to update.

Theorem 4. It is possible to process, in real time, a sequence of $R M Q$ commands in $O(\ell)$ space and in $O(\log \log \ell)$ time with high probability, for all operations except Value, which requires $O(\sqrt{\ell})$ time with high probability.

Proof. First note that the Value command is not used in the de-amortized transfer procedure described in Lemma 6. Thus guaranteeing that the overhead per command will be only $O(\log \log \ell)$ time, once the statement of the Theorem is established.

One important consideration is to reduce the high probability time of the hash-table to $O(\log \log \ell)$ instead of $O(\log \ell)$. For this goal we modify the separate chaining to the 2 -way chaining approach proposed by Azar, Broder, Karlin, and Upfal [1999], also with a maximum load factor of $50 \%$. 
We can now analyze the Van Emde Boas tree (VEB). This data structure is used as in Theorem 3 to store the minimum values of each set. Hence the underlying universe are the positions over $A$. Since this structure uses linear space in the universe size this would yield $O(n)$ space. However in this case we can use the hash-table to reduce the position range and thus the required space becomes $O(\ell)$. Note that the reduced positions are also integers and we can thus correctly use this data structure.

Given that the time to compute a predecessor with this data structure is $O(\log \log \ell)$ this then implies this bound for the RMQ operations except Value. For this operation we have two caveats. First the binary search over the values in the stack $S$ still requires $O(\log \ell)$ time. Second the Union operations in Algorithm 5 implies that it is necessary to remove elements from the VEB tree. This is not a problem for the Mark operation, Algorithm [6] because a single removal in this tree also requires $O(\log \log \ell)$ time. The issue for Value is that it may perform several such operations. In particular when $d$ elements are removed from the stack it requires $O(d \log \log \ell)$ time. Recall the example in the proof of Theorem 3, where several union operations where executed to produce the set $\{1\} \cup\{2\} \cup \emptyset \cup\{4,5,6\} \cup\{7\}$. In that Theorem this was done automatically by modifying $k$, but in this case it is necessary to actually remove the elements 2, 3, 4 and 7 from the VEB tree. Note that the element 3 is the representative of the empty set. Even though it is not active it was still in the VEB tree.

This consists in removing from the VEB tree all the elements that are larger than 1 . The VEB tree does not have a native operation for this process. Hence we have thus far assumed that this was obtained by iterating the delete operation. Still it is possible to implement this bulk delete operation directly within the structure, much like it can be done over binary search trees. In essence the procedure is to directly mark the necessary first level structures as empty and then do a double recursion, which is usually strictly avoided in this data structure. Given a variable $u$ that identifies the logarithm of the universe size as $\ell=2^{u}$, this yields the following time recursion $T(u)=2^{u / 2}+2 T(u / 2)$. Note that $2^{u / 2}=\sqrt{\ell}$ is the number of structures that exist in the first level, and potentially need to be modified. This recursion is bounded by $O\left(2^{u / 2}\right)=O(\sqrt{\ell})$.

As a final remark about this last result note that the time bound for the Value command is also $O(\log \log \ell)$ amortized, only the high probability bound is $O(\sqrt{\ell})$. This is because the iterated deletion bound $O(d \log \log \ell)$ that we mentioned in the proof does amortize to $O(\log \log \ell)$ and for each instance of the Value command we can choose between $O(d \log \log \ell)$ and $O(\sqrt{\ell})$.

This closes the theoretical analysis of the data structure. Further discussion is given in Section 6 .

\section{Experimental}

Let us now focus on testing the performance of this structure experimentally. We implemented the data structure that is described in Theorem 2. We also designed a generator that produces random sequences of RMQ commands. In these generated sequences the array $A$ contained $2^{28}$ integers, i.e., $n=2^{28}$. Each integer was chosen uniformly between 0 and $2^{30}-1$, with the arc4random_uniform 
function!

We first implemented the version of our Algorithm described in Section2, i.e., without using a hash table nor the transfer process. We refer to the prototype as the vanilla version and use the letter $\mathrm{V}$ to refer to it in our tables. We also implemented the version described in Theorem 2, which includes a hash table and requires a transfer process. We use the label T2 to refer to this prototype.

For a baseline comparison we used the ST-RMQ-CON algorithm by Alzamel. Charalampopoulos. Iliopoulos, a [2018]. We obtained the implementation from their github repository https://github.com/solonas 13/rmqo

Our RMQ command sequence generator proceeds as follows. First it generates $n=2^{28}$ integers uniformly between 0 and $2^{30}-1$. Then it chooses a position to Mark, uniformly among the $n$ positions available. This process is repeated $q$ times. Note that the choices are made with repetition, therefore the same position can be chosen several times. Each marked position in turn will force a query command. All query intervals have the same length $l=j-i+1$. Under these conditions it is easy to verify that the expected number of open positions at a given time is $l \times q / n$ and the actual number should be highly concentrated around this value. Hence we assume that this value corresponds to our $\ell$ parameter and therefore determine $l$ as $\ell \times n / q$.

The tests were performed on a 64 bit machine, running Linux mem 4.19.0-12, which contained 32 cores in Intel $(R)$ Xeon (R) CPU E7- 4830 @ $2.13 \mathrm{GHz}$ CPUs. The system has $256 G b$ of RAM and of swap. Our prototypes were compile with gcc 8.3.0 and the baseline prototype with $\mathrm{g}^{++}$. All prototypes are compiled with -03 . We measure the average execution time by command and the peak memory used by the prototypes. These values were both obtained with the system time command. These results are show in table 2 and 3 ,

The results show that our prototypes are very efficient. In terms of time both $\mathrm{V}$ and $\mathrm{T} 2$ obtain similar results, see Table 2, As expected T2 is slightly slower than $\mathrm{V}$, but in practice this different is less than a factor of 2 . The time performance of $\mathrm{B}$ is also very similar, in fact $\mathrm{V}$ and $\mathrm{T} 2$ are faster, which was not expected as $\mathrm{B}$ has $O(1)$ performance per operation and $\mathrm{V}$ and $\mathrm{T} 2$ have $O(\alpha(n))$. Even though in practice this difference was expected to be very small we were not expecting to obtain faster performance. This is possibly a consequence of the memory hierarchy as $\mathrm{B}$ works by keeping $A$ and all the queries in memory.

Concerning memory our prototypes also obtained very good performance, see Table 3, In particular we can clearly show a significant difference between using $O(q)$ and $O(\ell)$ extra performance. Consider for example $q=2^{26}$ and $\ell=2^{16}$. For these values $\mathrm{V}$ uses more than one gigabyte of memory, whereas $\mathrm{T} 2$ requires only $17 \mathrm{Mb}$, a very large difference. In general $\mathrm{T} 2$ uses less memory than $\mathrm{V}$, except when $q$ and $\ell$ become similar. For example when $q=\ell=2^{26}$ $\mathrm{V}$ use around one gigabyte of memory, whereas T2 requires three, but this is expected. Up to a given fixed factor. The baseline B requires much more memory as it stores more items in memory. Namely a compacted version of the array $A$ and the solutions to all of the queries. Our prototypes $\mathrm{V}$ and $\mathrm{T} 2$ do not store query solutions. Instead whenever a query is computed its value is written to a volatile variable. This guarantees that all the necessary computation is performed, instead of optimized away by the compiler. However it also means that previous solutions are overwritten by newer results. We deemed this solution as adequate for an online algorithm, which in practice will

\footnotetext{
${ }^{1}$ https://github.com/freedesktop/libbsd
} 


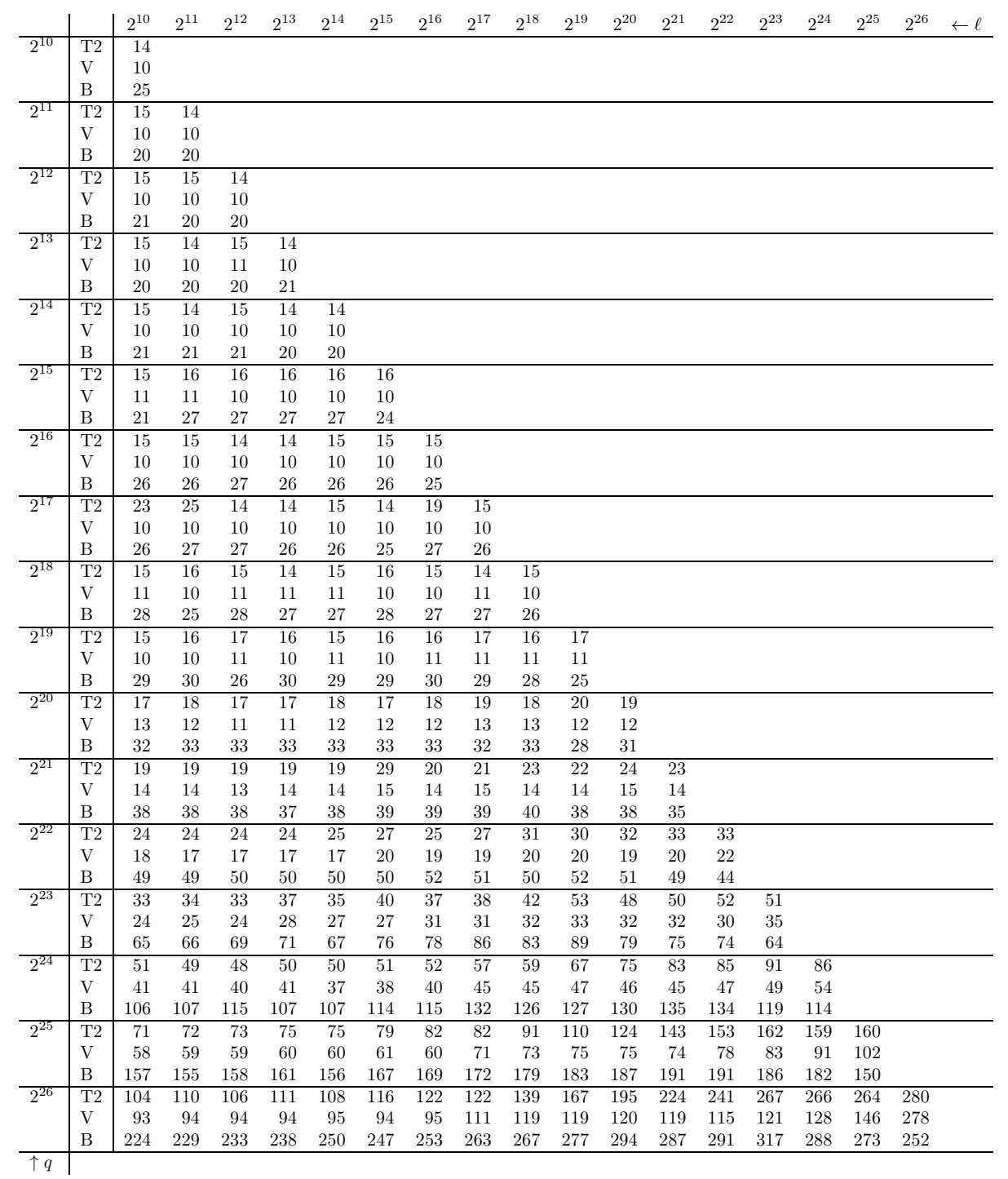

Table 2: Execution time per command in nano seconds. The values are obtained by dividing total execution time by $n+q$. 


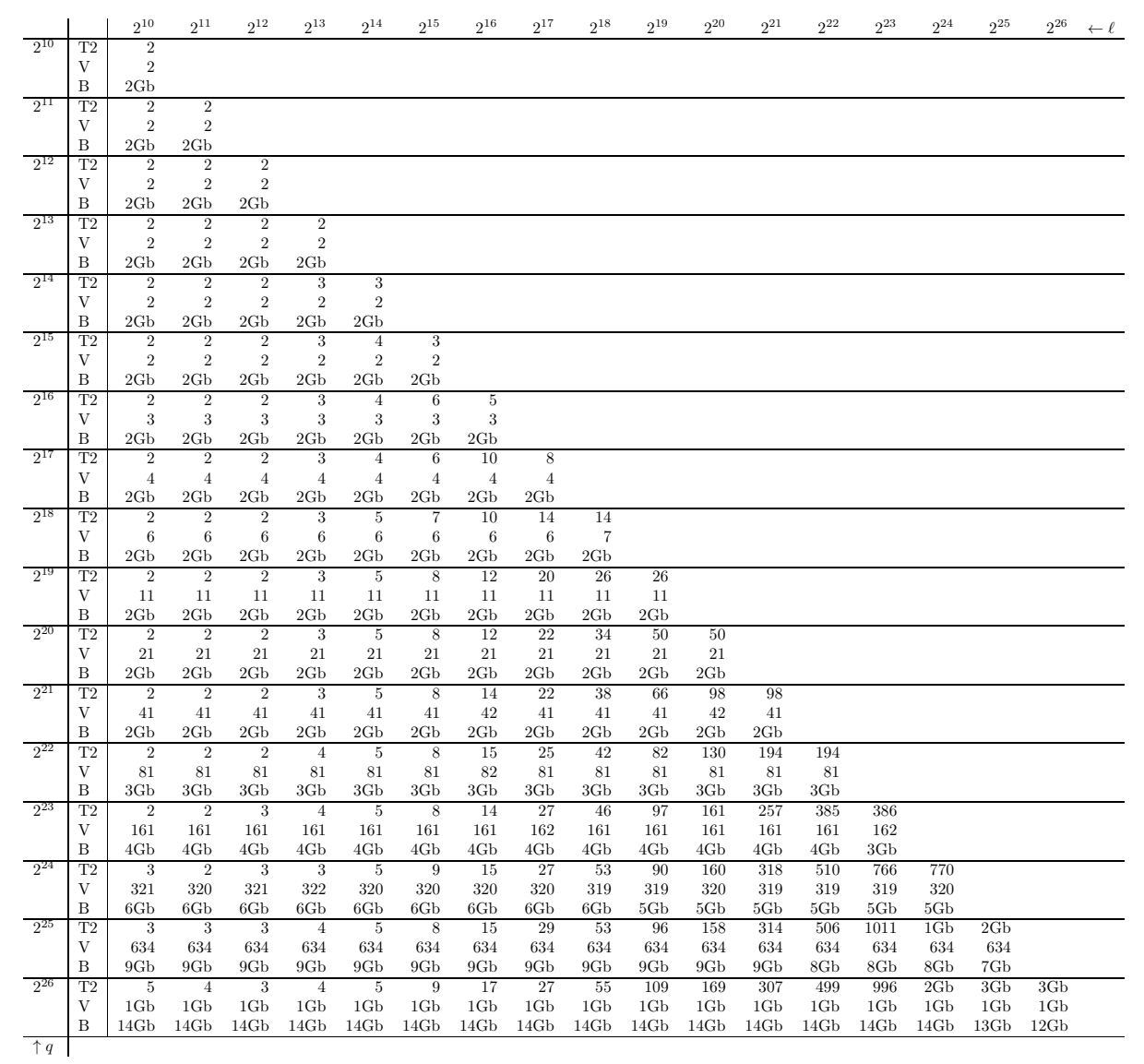

Table 3: Total memory peak in Megabytes, or in Gygabytes when indicated by $\mathrm{Gb}$. 
most likely pass its results to a calling process. Moreover storing the query solutions would bound the experimental results to $\Omega(q)$ space, thus not being a fair test of $O(\ell)$ space.

\section{Related Work}

The Range Minimum Query problem has been exhaustively studied. This problem was shown to be linearly equivalent to the Lowest Common Ancestor problem in a static tree by Gabow, Bentley, and Tarian |1984|. A recent perspective on this result was given by Bender and Farach-Colton [2000]. The first major solution to the LCA problem, by Berkman and Vishkin [1993], obtained $O(\alpha(n))$ time, using Union-Find data structures. Similarly to our data structure. In fact this initial result was a fundamental inspiration for the data structure we propose in this paper. A constant time solution was proposed by Harel and Tarian [1984]. A simplified algorithm was proposed by Schieber and Vishkin [1988]. A simplified exposition of these algorithms, and linear equivalence reductions, was given by Bender and Farach-Colton [2000].

Even though these algorithms were simpler to understand and implement they still required $O(n)$ space to store auxiliary data structures, such as Cartesian trees. Moreover the constants associated with these data structures were large, limiting the practical application of these algorithms. To improve this limitation direct optimal direct algorithms for RMQ were proposed by Fischer and Heun [2006]. The authors also showed that their proposal improved previous results by a factor of two. However they also observed that for several common problem sizes, asymptotically slower variants obtained better performance. Hence a practical approach, that obtained a 5 time speedup, was proposed by Ilie, Navarro, and Tinta [2010]. Their approach was geared towards the Longest Common Extension on strings and leveraged the use its average value to.

A line of research directed by an approach that focused on reducing constants by using succinct and compressed representations was initiated by Sadakane 2007a and successively improved by Sadakane [2007b], Sadakane and Navarro [2010] and Fischer and Heun 2011]. The last authors provide a systematic comparison of the different results up to 2011. Their solution provided an $2 n+o(n)$ bits data structure the answers queries in $O(1)$ time.

Still several engineering techniques can be used obtain more practical efficient solutions. An initial technique was proposed by Grossi and Ottaviano [2013]. A simplification implemented by Ferrada and Navarro 2017] used 2.1n bits and answered queries in 1 to 3 microseconds per query. Another proposal by Baumstark, Gog. Heuer, and Labeit [2017] obtained around a 1 microsecond per query (timings vary depending on query parameters) on an single core of the Intel Xeon E5-4640 CPU.

A new approach was proposed by Alzamel. Charalampopoulos. Iliopoulos, and Pissis [2018] where no index data structure was created by a preprocessing step. Instead all the RMQs are batched together and solved in $n+O(q)$ time and $O(q)$ space. This space was used to store a contracted version of the input array $A$ and the solutions to the queries. This is essentially the approach we follow in this paper. Therefore in Table 2 we independently verify their query times in the nanoseconds. Also table 3 reports the memory requirements of their structure. 
In a recent result Kowalski and Grabowski 2018 proposed an heuristic idea, without constant worst case time and a hybrid variation with $O(1)$ time and $3 n$ bits. Their best result obtains competitive results against existing solutions, except possibly for small queries. Their results show query times essentially equal to ours and the algorithm of Alzamel, Charalampopoulos, Iliopoulos, and Pissis 2018 for large queries, but they also obtain 10 times slower performance for small queries.

For completion we also include references to the data structures we used, or mentioned, in our approach.

The technique by Briggs and Torczon 1993] provides a way to use memory without the need to initialize it. Moreover each time a given memory position needs to be used for the first time it requires only $O(1)$ time to register this change. The trade-off with this data structure is that it triples the space requirements. Since, for now, we do not have an implementation of Lemma 6, the claimed result can use this technique, also explained by Bentley [2016] and Aho and Hopcroft [1974]. For our particular implementation this can be overcome. For the destination structure is not a problem because we can assume that the whole merge process includes the time for the initial clean-up, all within $\left(a_{1}+a_{2}\right) / 2$ as explained in Lemma 6 . Only the active structure requires some more forethought. In essence when the merge processes starts and we start using an active structure that supports $\left(a_{1}+a_{2}\right) / 2$ elements it is a good time to start cleaning a piece of memory that supports $\left(a_{1}+a_{2}+c_{1}+c_{2}\right) / 2$ elements, as this will be the number of elements of the future active structure. We will start using this structure when the current merge finishes. Since this number of elements is at most $a_{1}+a_{2}$ it is possible to finish the clean-up when at most $\left(a_{1}+a_{2}\right) / 2$ operations have executed, by cleaning two element positions in each operation.

The Union-Find data structure is a fundamental piece of our solution. The original proposal to represent disjoint sets that can support the Union and Find operations was by Galler and Fisher 1964]. Their complexity was bounded by $O\left(\log ^{*}(n)\right)$ amortized time per operation by Hopcroft and Ullman [1973|. The analysis of the time bound was later refined to $O(\alpha(n))$ by Tarian and van Leeuwen 1984. Lower bound analysis guarantees that these bounds are optimal Tarjan [1979] and Fredman and Saks 1989. However in the case where the sequence of operations is known a priori it is possible to obtain $O(1)$ amortized time per operation, as shown by Gabow and Tarian [1985]. An exhaustive survey was given by Galil and Italiano 1991|. An elementary description of this data structure was provided by Cormen, Leiserson, Rivest, and Stein [2009] and Sedgewick and Wayne 2011.

Hash tables date back to the origin of computers. A history on the subject and the first theoretical analysis was given by Knuth [1963]. This analysis established constant expect time bound. The high probability bound of separate chaining can be derived from balls and bins model, see Mitzenmacher and Upfal [2017]. Actually a better bound was obtained by Gonnet 1981|. The 2-way chaining hash-table was proposed by Azar. Broder, Karlin, and Upfal 1999], which also established its constant expected time and high probability bound.

Exponential searches where proposed by Bentley and Yao [1976] and Baeza-Yates and Salinger [2010] and can be used to speed-up the binary search algorithm when the desired element is close to the beginning or end of a list. For an introduction to binary search see Cormen et al. 2009. 
The data structure by Boas. Kaas, and Ziilstra 1976] provides support for Predecessor queries over integers in $O(\log \log n)$ time, by recursively dividing a tree along its medium height. For an elementary description, which requires less space was given by Cormen. Leiserson. Rivest, and Stein [2009]. The yfast trie data structure was proposed by Willard [1983] to reduce the large space requirements of the Van Emde Boas tree. This data structure obtains the $O(\log \log n)$ time bound, only that amortized. For this reason we did not considered it in Theorem 4. Also in the process the this result describes $\mathrm{x}$-fast tries.

\section{Discussion and Conclusion}

We can now discuss our results in context. In this paper we started by defining a set of commands that can be used to form sequences. Although these commands are fairly limited they can still be used for several important applications. First notice that if we are given a list of $(i, j)$ RMQs we can reduce them to the classical context. This can be achieved with two hash tables. In the first table store the queries indexed by $i$ and on the second by $j$. We use the first table to issue Mark commands and the second to issue Query commands. This requires some overhead but it allows our approach to be used to solve classical RMQ problems. In particular it will significantly increase the memory requirements, as occurs in Table 3 between $\mathrm{T} 2$ and B.

Our data structures can be used in online and real-time applications. Note in particular we can use our commands to maintain the marked positions in a sliding window fashion. Meaning that at any instant we can issue Query commands for any of the previous $\ell$ positions. The extremely small memory requirements of our approach makes our data structure suitable to be used in routers, switches or in embedded computation devices with low memory and CPU resources.

The simplest configuration of our data structure consists of a stack combined with a Union-Find data structure. For this structure we can formally prove that our procedures correctly compute the desired result, Theorem 1. We then focused on obtaining the data structure configuration that yielded the best performance. We started by obtaining $O(\alpha(n))$ amortized time and $O(q)$ space, see Theorem 2 This result is in theory slower than the result by Alzamel. Charalampopoulos. Iliopoulos, and Pissis 2018], which obtained $O(1)$ amortized query time. We compared experimentally these approaches in Section 4. The results showed that out approach was competitive, both in terms of time and space, our prototype $\mathrm{V}$ was actually faster than the prototype B by Alzamel et al. [2018]. We also showed that it was possible for our data structure to obtained $O(1)$ amortized query time (Corolary 1), mostly for theoretical competitiveness. We did not implement this solution.

We described how to reduce the space requirements down to $O(\ell)$, by transferring information among structures and discarding structures that became full, see Lemma 6. In theory this obtained the same $O(\alpha(n))$ amortized time but significantly reduced space requirements. We also implemented this version of the data structure. In practice the time penalty was less than a 2 factor. Moreover, for some configurations, the memory reduction was considerable, see Table 3. 
Lastly we focused on obtaining real time performance. We obtained a high probability bound of $O(\log n)$ amortized time per query, see Theorem 3 This bound guarantees real time performance. We then investigated alternatives to reduce this time bound to $O(\log \log n)$. We proposed two solutions. In one case we considered approximate queries, thus reducing the necessary amount of active positions to $O(\log n)$. In the other case we used the Van Emde Boas tree, which provided a $O(\log \log n)$ high probability time bound for all commands except Value, see Theorem 4. In this later configuration the Value command actually obtained an $O(\sqrt{\ell})$ bound, which is large, but the corresponding amortized value is only $O(\log \log n)$.

\section{Acknowledgements}

The work reported in this article was supported by national funds through Fundação para a Ciência e a Tecnologia (FCT) with reference UIDB/50021/2020 and project NGPHYLO PTDC/CCI-BIO/29676/2017.

\section{References}

Maxime Crochemore and Luís M.S. Russo. Cartesian and Lyndon trees. Theoretical Computer Science, 806:1-9, feb 2020.

Harold N. Gabow and Robert Endre Tarjan. A linear-time algorithm for a special case of disjoint set union. Journal of Computer and System Sciences, 30(2):209-221, apr 1985.

Preston Briggs and Linda Torczon. An efficient representation for sparse sets. ACM Letters on Programming Languages and Systems, 2(1-4):59-69, mar 1993.

Michael Mitzenmacher and Eli Upfal. Probability and computing: Randomization and probabilistic techniques in algorithms and data analysis. Cambridge university press, 2017.

Robert E. Tarjan and Jan van Leeuwen. Worst-case analysis of set union algorithms. Journal of the ACM, 31(2):245-281, mar 1984.

Yossi Azar, Andrei Z. Broder, Anna R. Karlin, and Eli Upfal. Balanced allocations. SIAM Journal on Computing, 29(1):180-200, jan 1999.

Mai Alzamel, Panagiotis Charalampopoulos, Costas S. Iliopoulos, and Solon P. Pissis. How to answer a small batch of RMQs or LCA queries in practice. In Lecture Notes in Computer Science, pages 343-355. Springer International Publishing, 2018.

Harold N. Gabow, Jon Louis Bentley, and Robert E. Tarjan. Scaling and related techniques for geometry problems. In Proceedings of the sixteenth annual ACM symposium on Theory of computing - STOC 84. ACM Press, 1984.

Michael A. Bender and Martín Farach-Colton. The LCA problem revisited. In Lecture Notes in Computer Science, pages 88-94. Springer Berlin Heidelberg, 2000. 
Omer Berkman and Uzi Vishkin. Recursive star-tree parallel data structure. SIAM Journal on Computing, 22(2):221-242, apr 1993.

Dov Harel and Robert Endre Tarjan. Fast algorithms for finding nearest common ancestors. SIAM Journal on Computing, 13(2):338-355, may 1984.

Baruch Schieber and Uzi Vishkin. On finding lowest common ancestors: Simplification and parallelization. SIAM Journal on Computing, 17(6):1253-1262, $\operatorname{dec} 1988$.

Johannes Fischer and Volker Heun. Theoretical and practical improvements on the RMQ-problem, with applications to LCA and LCE. In Combinatorial Pattern Matching, pages 36-48. Springer Berlin Heidelberg, 2006.

Lucian Ilie, Gonzalo Navarro, and Liviu Tinta. The longest common extension problem revisited and applications to approximate string searching. Journal of Discrete Algorithms, 8(4):418-428, dec 2010.

Kunihiko Sadakane. Compressed suffix trees with full functionality. Theory of Computing Systems, 41(4):589-607, feb 2007a.

Kunihiko Sadakane. Succinct data structures for flexible text retrieval systems. Journal of Discrete Algorithms, 5(1):12-22, mar 2007b.

Kunihiko Sadakane and Gonzalo Navarro. Fully-functional succinct trees. In Proceedings of the Twenty-First Annual ACM-SIAM Symposium on Discrete Algorithms. Society for Industrial and Applied Mathematics, jan 2010.

Johannes Fischer and Volker Heun. Space-efficient preprocessing schemes for range minimum queries on static arrays. SIAM Journal on Computing, 40(2): 465-492, jan 2011.

Roberto Grossi and Giuseppe Ottaviano. Design of practical succinct data structures for large data collections. In Experimental Algorithms, pages 5-17. Springer Berlin Heidelberg, 2013.

Héctor Ferrada and Gonzalo Navarro. Improved range minimum queries. Journal of Discrete Algorithms, 43:72-80, mar 2017.

Niklas Baumstark, Simon Gog, Tobias Heuer, and Julian Labeit. Practical Range Minimum Queries Revisited. In 16th International Symposium on Experimental Algorithms (SEA 2017), volume 75 of Leibniz International Proceedings in Informatics (LIPIcs), pages 12:1-12:16, Dagstuhl, Germany, 2017. Schloss Dagstuhl-Leibniz-Zentrum fuer Informatik. ISBN 978-3-95977-036-1.

Tomasz M. Kowalski and Szymon Grabowski. Faster range minimum queries. Software: Practice and Experience, 48(11):2043-2060, 2018.

Jon Bentley. Programming pearls. Addison-Wesley Professional, 2016.

Alfred V Aho and John E Hopcroft. The design and analysis of computer algorithms. Pearson Education India, 1974.

Bernard A. Galler and Michael J. Fisher. An improved equivalence algorithm. Communications of the ACM, 7(5):301-303, may 1964. 
J. E. Hopcroft and J. D. Ullman. Set merging algorithms. SIAM Journal on Computing, 2(4):294-303, dec 1973.

Robert Endre Tarjan. A class of algorithms which require nonlinear time to maintain disjoint sets. Journal of Computer and System Sciences, 18(2):110127, apr 1979 .

M. Fredman and M. Saks. The cell probe complexity of dynamic data structures. In Proceedings of the twenty-first annual ACM symposium on Theory of computing - STOC 89. ACM Press, 1989.

Zvi Galil and Giuseppe F. Italiano. Data structures and algorithms for disjoint set union problems. ACM Computing Surveys, 23(3):319-344, sep 1991.

Thomas H Cormen, Charles E Leiserson, Ronald L Rivest, and Clifford Stein. Introduction to algorithms. MIT press, 2009.

Robert Sedgewick and Kevin Wayne. Algorithms. Addison-wesley professional, 2011.

Don Knuth. Notes on" open" addressing. 1963.

Gaston H. Gonnet. Expected length of the longest probe sequence in hash code searching. Journal of the ACM, 28(2):289-304, apr 1981.

Jon Louis Bentley and Andrew Chi-Chih Yao. An almost optimal algorithm for unbounded searching. Information Processing Letters, 5(3):82 - 87, 1976. ISSN 0020-0190.

Ricardo Baeza-Yates and Alejandro Salinger. Fast intersection algorithms for sorted sequences. In Algorithms and Applications, pages 45-61. Springer Berlin Heidelberg, 2010.

P. Emde Boas, R. Kaas, and E. Zijlstra. Design and implementation of an efficient priority queue. Mathematical Systems Theory, 10(1):99-127, dec 1976.

Dan E. Willard. Log-logarithmic worst-case range queries are possible in space $\Theta(\mathrm{n})$. Information Processing Letters, 17(2):81-84, aug 1983. 